ACCEPTED MANUSCRIPT

\title{
Bond-orientational order and Frank's constant in two-dimensional colloidal hard spheres
}

To cite this article before publication: Alice L. Thorneywork et al 2018 J. Phys.: Condens. Matter in press https://doi.org/10.1088/1361$\underline{648 X / a a a b 31}$

\author{
Manuscript version: Accepted Manuscript \\ Accepted Manuscript is "the version of the article accepted for publication including all changes made as a result of the peer review process, \\ and which may also include the addition to the article by IOP Publishing of a header, an article ID, a cover sheet and/or an 'Accepted \\ Manuscript' watermark, but excluding any other editing, typesetting or other changes made by IOP Publishing and/or its licensors" \\ This Accepted Manuscript is @ 2018 IOP Publishing Ltd.
}

During the embargo period (the 12 month period from the publication of the Version of Record of this article), the Accepted Manuscript is fully protected by copyright and cannot be reused or reposted elsewhere.

As the Version of Record of this article is going to be / has been published on a subscription basis, this Accepted Manuscript is available for reuse under a CC BY-NC-ND 3.0 licence after the 12 month embargo period.

After the embargo period, everyone is permitted to use copy and redistribute this article for non-commercial purposes only, provided that they adhere to all the terms of the licence https://creativecommons.org/licences/by-nc-nd/3.0

Although reasonable endeavours have been taken to obtain all necessary permissions from third parties to include their copyrighted content within this article, their full citation and copyright line may not be present in this Accepted Manuscript version. Before using any content from this article, please refer to the Version of Record on IOPscience once published for full citation and copyright details, as permissions will likely be required. All third party content is fully copyright protected, unless specifically stated otherwise in the figure caption in the Version of Record.

View the article online for updates and enhancements. 


\title{
Bond-orientational order and Frank's constant in two-dimensional colloidal hard spheres
}

\author{
Alice L. Thorneywork ${ }^{1,2}$, Joshua L. Abbott ${ }^{1}$, Dirk G. A. L. \\ Aarts $^{1}$, Peter Keim ${ }^{3}$ and Roel P. A. Dullens ${ }^{1}$ \\ 1 Department of Chemistry, Physical and Theoretical Chemistry Laboratory, \\ University of Oxford, South Parks Road, Oxford, OX1 3QZ, United Kingdom \\ ${ }^{2}$ Cavendish Laboratory, University of Cambridge, Cambridge CB3 0HE, United \\ Kingdom \\ ${ }^{3}$ Department of Physics, University of Konstanz, Konstanz 78464, Germany
}

\begin{abstract}
Recently, the full phase behaviour of two-dimensional colloidal hard spheres was experimentally established, and found to involve a first order liquid to hexatic transition and a continuous hexatic to crystal transition [A. L. Thorneywork et al., Phys. Rev. Lett. 118, 158001 (2017)]. Here, we expand upon this work by considering the behaviour of the bond-orientational correlation time and Frank's constant in the region of these phase transitions. We also consider the excess entropy, as calculated from the radial distribution functions, for a wide range of area fractions covering the liquid, hexatic and crystal phases. In all cases, the behaviour of these quantities further corroborates the previously reported melting scenario of two-dimensional colloidal hard spheres.
\end{abstract}

\section{Introduction}

The spatial dimensionality of a system can have a profound effect upon its physical properties. This may be observed in both structural and dynamic phenomena, with examples including the absence of long-range translational order in a two-dimensional (2D) crystal [1] and the differing diffusive behaviour seen in one and two dimensions $[2,3]$. Another notable and widely studied example is the melting transition in 2D. Here, unlike melting in 3D [4], it is proposed that an additional 'hexatic' phase exists between the $2 \mathrm{D}$ liquid and crystalline phases. This results in melting via two consecutive phase transitions, a scenario/described by the KTHNY theory from Kosterlitz, Thouless, Halperin, Nelson and Young $[5,6,7,8]$.

More specifically, KTHNY theory links the melting of 2D materials to the unbinding of topological defects. The three key types of defects - dislocation pairs, dislocations and disclinations - all consist of five- or seven-fold coordinated particles, and are illustrated in Fig. 1. Crystalline states in 2D possess quasi-long-ranged translational order and long-ranged bond-orientational order and the only type of defect consistent with this is a dislocation pair, which is composed of two five-fold and two seven-fold 


\section{Bond-orientational order and Frank's constant in two-dimensional colloidal hard spheres2}

(a)

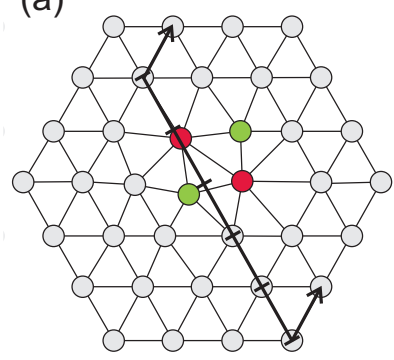

Crystal (b)

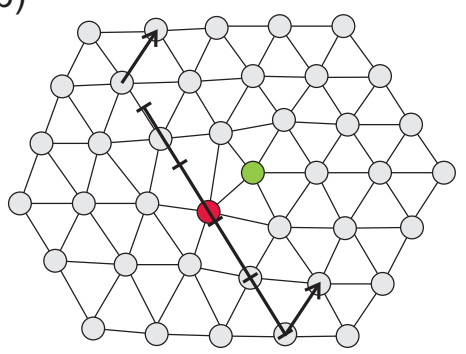

Hexatic (c)

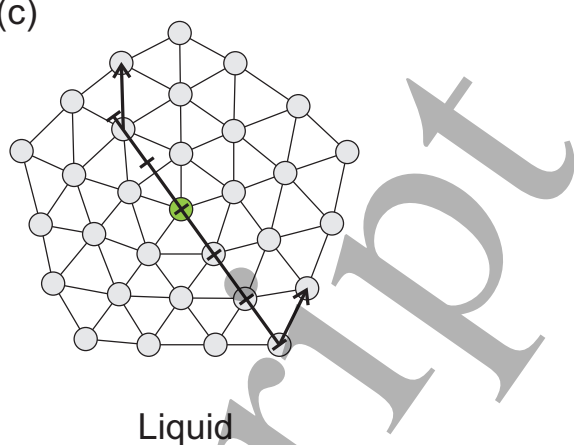

Figure 1. An illustration of the defects seen in the melting of 2D c̀rystals: (a) a dislocation pair ; (b) a dislocation; and (c) a 5-fold disclination. Particles coloured red are those with seven nearest neighbours and particles coloured green are those with five nearest neighbours. Arrows indicate the bond-orientation on each side of the defect and lines mark the particle positions expected for systems with translational order.

coordinated particles (see Fig. 1a). Clearly shown here is that the presence of a bound dislocation pair in a crystal does not disrupt the translational or bond-orientational order of the crystal. The softening of the shear elasticity of the crystal in the vicinity of the crystal-hexatic transition can be linked to the presence of these dislocation pairs and is described by a recursion relation: as more dislocation pairs appear the crystal becomes increasingly soft, which in turn increases the probability of the creation of new dislocation pairs, which further softens the crystal. At the crystal-hexatic transition, bound dislocation pairs unbind into isolated dislocations as a result of which the shear elasticity disappears $[6,7,8]$. A dislocation, consisting of a five-fold and a seven-fold coordinated particle, is shown in Fig. 1b, and leads to a loss of translational order but the retention of bond-orientational order. The resulting hexatic phase is therefore characterised by short-ranged translational order and quasi-long-ranged six-fold bondorientational order. The second step of melting to an isotropic fluid is associated with the unbinding of dislocations to form isolated five- and seven-fold disclinations (see Fig. 1c), which disrupt the system with respect to both the translational and bondorientational order. The resulting liquid phase thus has only short-ranged translational and short-ranged bond-orientătional order.

The spatial range of the bond-orientational order is quantified by the bondorientational correlation function $[9,10]$

$$
2 \quad g_{6}(r)=\operatorname{Re}\left\langle\frac{\psi_{6, i}(r) \cdot \psi_{6, i}^{*}(0)}{\left|\psi_{6, i}\right|^{2}}\right\rangle \text {, }
$$

with the sixfold bond-orientational order parameter, $\psi_{6, i}$, defined as [10]

$$
\psi_{6, i}(t)=\frac{1}{n} \sum_{j=1}^{n} \exp \left(i 6 \theta_{i j}(t)\right) .
$$

Here $n$ is the number of nearest neighbours and $\theta_{i j}$ is the angle between the vector connecting the central particle, $i$, with neighbouring particles, $j$, and a fixed reference 
axis. At large $r g_{6}(r)$ attains a constant value in the crystal, but decays algebraically in the hexatic phase, with $g_{6}(r) \sim r^{-1 / 4}$ at the transition between the hexatic and liquid $[7,11]$. In the liquid, the short-ranged bond-orientational order leads to an exponential decay of $g_{6}(r)$.

Alternatively, dynamic criteria can be used to distinguish between the liquid, hexatic and crystal states [12], and these are particularly useful for the consideration of experimental systems imaged over a finite distance for a long time. Here, the analogous expression to Eq. (1) considers the variation in the value of $g_{6}$ with time, as $[10,12,13]$

$$
g_{6}(t)=\operatorname{Re}\left\langle\frac{\psi_{6, i}(t) \cdot \psi_{6, i}^{*}(0)}{\left|\psi_{6, i}\right|^{2}}\right\rangle,
$$

with $\langle\ldots\rangle$ denoting an average taken over all particles and time origins. For long times, $g_{6}(t)$ tends towards a constant for the crystal phase whilst in the hexatic phase, $g_{6}(t)$ decays algebraically as $g_{6}(t) \sim t^{-\eta_{6} / 2}$, where the exponent $0<\eta_{6}<1 / 4[7,10]$. Hence, $g_{6}(t) \sim t^{-1 / 8}$ at the hexatic-liquid transition $[10,12]$. In the liquid phase $g_{6}(t)$ decays exponentially as $g_{6}(t) \sim \exp \left(-t / \tau_{6}\right)$ with $\tau_{6}$ a characteristic bond-orientational correlation time. The exponent $\eta_{6}$ is directly related to the Frank elastic constant $F_{A}$ as $[7,11]$

$$
\eta_{6}=\frac{18 k_{B} T}{\pi F_{A}}
$$

with $k_{B}$ Boltzmann's constant and $T$ the temperature. Frank's constant is an effective stiffness of the bond-orientational field, or, in short, an orientational stiffness $[11,14]$. Because $\eta_{6}=1 / 4$ at the liquid-hexatic transition [7, 11], the value of Frank's constant at this transition is $F_{A} /\left(k_{B} T\right)=72 / \pi$.

The nature of the two-dimensional melting transition has been extensively studied in computer simulations ever since the introduction of the problem by Alder and Wainwright in 1962 [15]. While it is beyond the scope of this article to provide a comprehensive review of all of these simulations studies, we wish to highlight some more recent advances considering factors known to alter the melting scenario. Firstly, the dependence on interaction potential has been studied for particles with a short-range attraction between them [16] and, over particularly large length scales, for repulsive power-law interactions $[17,18]$, where the liquid-hexatic transition is found to change from first order to continuous with increasing softness of interaction [18]. For hard core interactions, the particle shape has been observed to effect both the nature of the phases and the order of the transitions [19]. Finally, particularly pertinent to experimental studies, are works considering the effect of pinned particles [20,21] and out of plane fluctuations [22], which are often found in quasi-2D experiments.

Experimentally, a major body of work considers the phase behaviour of monolayers of super-paramagnetic colloidal particles interacting via a soft potential $[9,11,12,23,24]$. For this system, it was established that melting takes place via two consecutive, continuous transitions with an intermediate hexatic phase. Furthermore, the specific heat [25] and elastic constants [11] around the transitions in this system have been 
reported and the effect of random pinning has been addressed [20]. Other experiments have also considered the melting behaviour of charge stabilised systems [26], sticky hard spheres [27, 28] and soft thermosensitive colloidal particles [13, 29].

The simplest interacting many-body system in 2D is a system of thermal hard disks as first studied with computer simulations in 1962 [15]. Inherent to the hard interactions, the phase diagram for hard disks is independent of temperature, and thus only dependent on the area fraction. For hard disks the nature of the melting transition has been the subject of a long and ongoing debate $[15,30,31,32,33,34]$, but recently large scale computer simulations reported two-step melting via a hexatic phase and a first order liquid-hexatic transition $[17,35]$. The two-step melting via a hexatic phase was then also experimentally established [36] by considering a colloidal monolayer in sedimentationdiffusion equilibrium. In particular, in the experiments it was found that melting of the crystal proceeds via a hexatic phase, with a first order liquid-hexatic transition and a coexistence region determined from the equation of state of $\phi \approx 0.68-0.70$. The hexatic phase was observed for $0.70<\phi<0.73$ and the continuous hexatic-crystal transition at $\phi \approx 0.73$. In this article, we expand upon these experimental results and consider the behaviour of the bond-orientational correlation time and Frank's constant upon approaching the liquid-hexatic and hexatic-crystal transition, respectively. In addition, we compute the excess entropy from the radial distribution functions for a wide range of area fractions covering the liquid, hexatic and crystals phases.

\section{Experimental methods}

The experiment has been introduced in [36], so only the essentials will be recapped here. We consider a tilted monolayer of melamine formalydehyde spheres with a hard-sphere

\section{(a)}

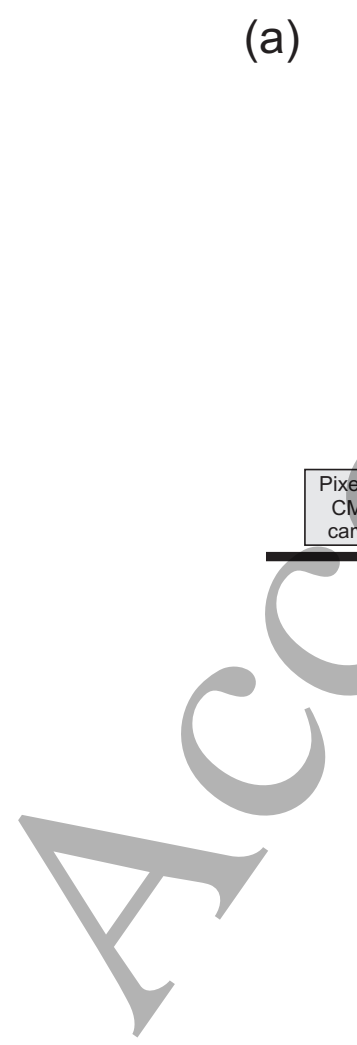

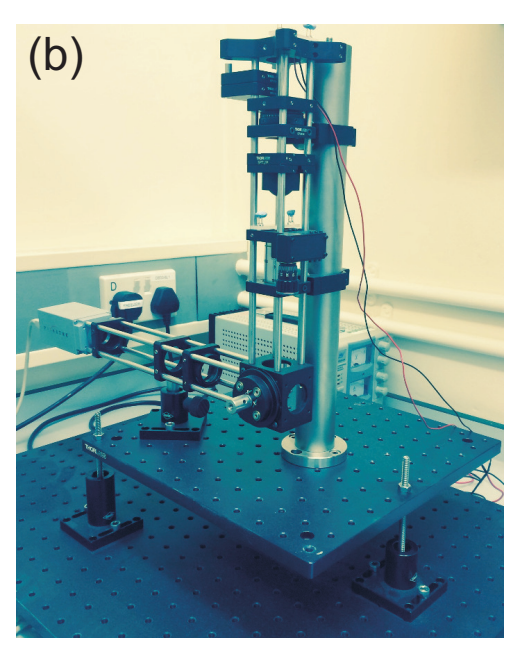

Figure 2. (a) A schematic of the custom-built tilting optical microscopy set-up. Inset: an image of the sample cell used: a Hellma quartz glass cell with internal width of $9 \mathrm{~mm}$, internal length of $20 \mathrm{~mm}$ and internal height of $200 \mu \mathrm{m}$. (b) A photograph of the the tilting microscopy set-up. 
diameter of $\sigma=2.79 \mu \mathrm{m}$ in a water-ethanol mixture. These particles form a monolayer at the base of a glass sample cell (quartz glass Hellma cell, see Fig. 2a inset) and are an excellent hard disk model system $[37,38]$. The samples are placed on a custom-built bright-field video microscope, which is then tilted by a small and variable angle, $\alpha$. In Fig. 2 we show a schematic (a) and a photograph (b) of the setup $\ddagger$. The system is imaged using a $20 \times$ objective with images recorded on a PixeLINK CMOS camera at a resolution of $1280 \times 1024$ pixels. The tilted monolayer is then left to equilibrate for many weeks to reach an in-plane sedimentation-diffusion equilibrium (see Fig. 1(a) of [36]). The system is imaged at a rate of one frame per sec for $2 \mathrm{~h}$, and standard particle tracking procedures [39] are used to obtain particle coordinates and tracks,

\section{Results and discussion}

\subsection{Bond-orientational order correlation time}

Upon approaching the hexatic phase from the liquid, it has been shown that the bondorientational correlation length diverges [11]. Consequently, it is also expected that the corresponding bond-orientational correlation time, $\tau_{6}$, diverges $[11,40,41]$. We determine $\tau_{6}$ from the bond-orientational correlation functions $g_{6}(t)$, shown in Fig. 3a for a tilt angle of $\alpha=0.25^{\circ}[36]$, via $g_{6}(t) \sim \exp \left(-t / \tau_{6}\right)$. The bond-orientational correlation time as a function of $\phi$ upon approaching the hexatic phase from the liquid phase is shown in Fig. 3b for data averaged over all six tilt angles. While at relatively low area fractions $\tau_{6}$ is about $10 \mathrm{~s}$, it sharply increases upon approaching the first order liquidhexatic phase transition at $\phi=0.68$ [36]. This is reminiscent of the critical fluctuations that diverge upon approaching a continuous transition, i.e. critical slowing down. In our case, however, this divergence is preempted by a weakly first order phase transition and hence the bond-orientational correlation time does not diverge. Nevertheless, we can still describe the onset of the divergence of $\tau_{6}$ for area fractions below the coexistence region using [11, 40, 41]

$$
\tau_{6} \sim \exp \left[\frac{a}{\left|1 / \phi-1 / \phi_{h}\right|^{\nu}}\right] .
$$

Here $a$ is a constant, $\phi_{h}$ the area fraction where the system becomes fully hexatic, and the exponent $\nu=1 / 2[11,40,41]$. As seen in Fig. 3b, the data are very well described by this expression and from the fit we obtain a value for $\phi_{h}=0.696$, which is in excellent agreement with the start of the hexatic phase at $\phi=0.70$ [36]. This is further corroborated by the behaviour of $\eta_{6}$ as a function of $\phi$ obtained using $g_{6}(t) \sim t^{-\eta_{6} / 2}$ $[10,36]$ and also shown in Fig. 3b. Here $\eta_{6}$ is close to zero at high area fractions $(\phi>0.73)$, but then starts to increase upon decreasing $\phi$ and reaches the value of $\eta_{6}=1 / 4$ at $\phi=0.70$. In other words, $\phi=0.70$ is the area fraction where the hexatic phase becomes unstable, consistent with the obtained value of $\phi_{h}=0.696$ from fitting Eq. (5) to our data. 
(a)

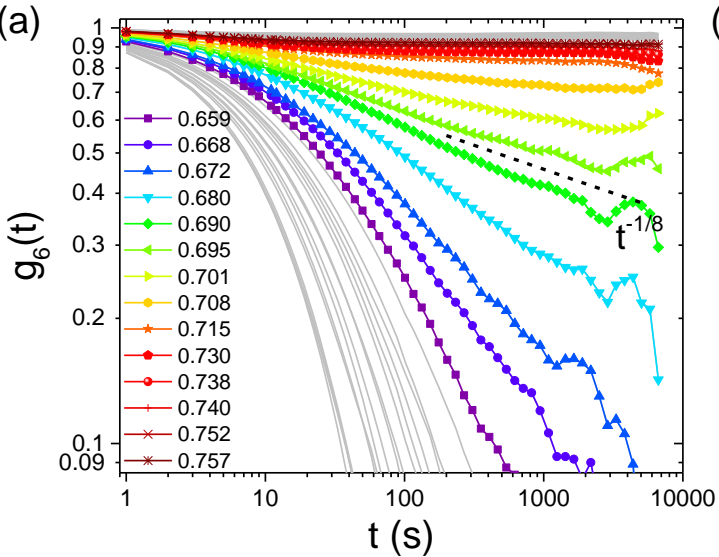

(b)

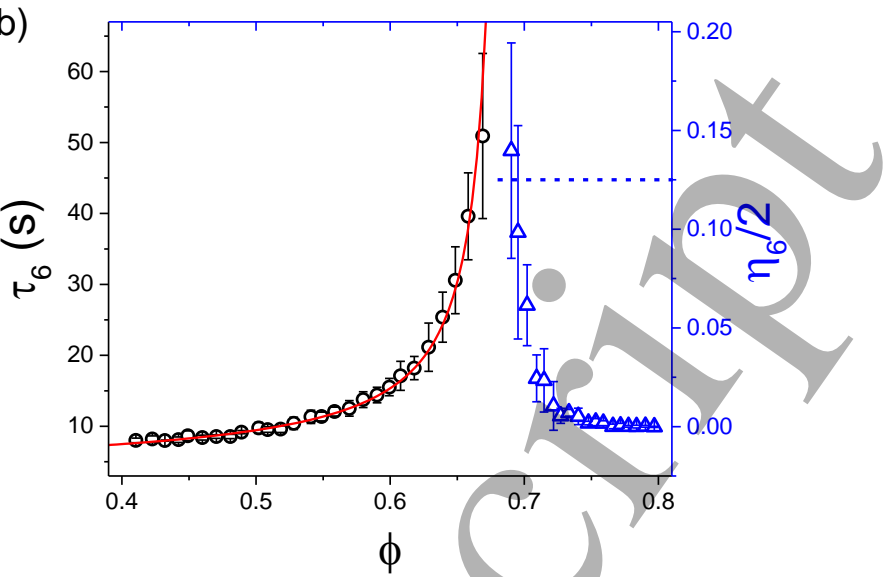

(c)

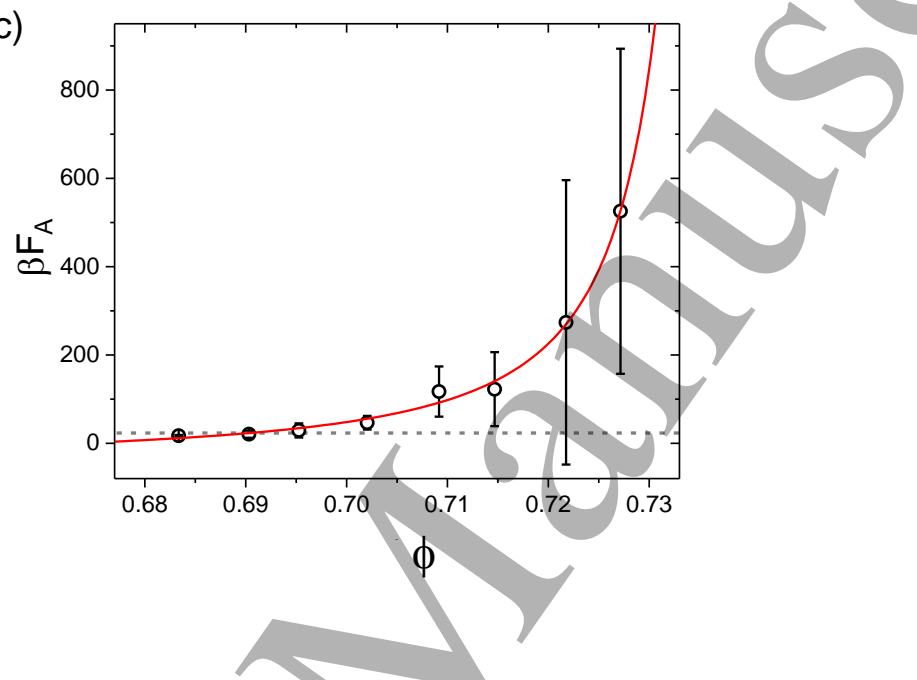

Figure 3. (a) The height-resolved bond-orientational correlation function in time, $g_{6}(t)$ [36], for the sample at $\alpha=0.25^{\circ}$ for all bins across the whole range of area fractions. Note that a legend is only provided for the data between $0.65<\phi<0.76$. (b) The bond-orientational time $\tau_{6}$ as a function of the area fraction $\phi$. The solid line is a fit according to Eq. (5). Also shown is the exponent $\eta_{6} / 2$ as a function of the area fraction, whose value of $1 / 8$ indicates the area fraction at which the hexatic phase becomes unstable (see dashed horizontal line). (c) Frank's constant, $F_{A}$, as a function of the area fraction $\phi$; the symbols are the experimental data and the solid line is a fit to the data according to Eq. (6). The horizontal straight line corresponds to the value of $72 / \pi$, i.e. below which the hexatic phase is unstable.

\subsection{Frank's constant}

Next, we consider the behaviour of Frank's constant, $F_{A}$, in the hexatic phase and, in particular, upon approaching the hexatic-crystal transition. Frank's constant is found directly from the exponent $\eta_{6}$ via Eq. (4) as $\beta F_{A}=18 / \pi \eta_{6}$ with $\beta=\left(k_{B} T\right)^{-1}$ and is plotted as a function of $\phi$ in Fig. 3c. At area fractions close to 0.70, i.e. where the hexatic phase starts, $\beta F_{A}$ is consistently close to the value of $72 / \pi$, i.e. where the hexatic phase is predicted to become unstable. As the area fraction increases, Frank's 
constant sharply increases and its divergence can be described by [11, 40, 41]

$$
\beta F_{A} \sim \exp \left[\frac{2 b}{\left|1 / \phi-1 / \phi_{c}\right|^{\bar{\nu}}}\right] .
$$

Here, $b$ is again a constant, $\phi_{c}$ the area fraction where the system becomes crystalline and the exponent $\bar{\nu}=0.36959$. Our data are well described by Eq. (6) and from the fit we obtain a value for $\phi_{c}$ of 0.733 . This is in good agreement with the location of hexatic-crystal transition at $\approx 0.73[36]$ - despite the relatively large error bars and the three fitting parameters - and corroborates the continuous nature of this transition.

\subsection{Excess configurational entropy}

Finally, we consider the excess configurational entropy, $S_{2}$, as it has been suggested that this can be used as an indicator for freezing in two dimensions [42]. In particular, $S_{2} / k_{B}$, the first term of the excess entropy arising from pair interactions, should attain a universal value of -4.5 at the freezing transition irrespective of the exact nature of the particle interactions [42]. For a $2 \mathrm{D}$ system, $S_{2}$, is defined by [43]

$$
S_{2}=-\frac{4 k_{B} \phi}{\sigma^{2}} \int_{0}^{\infty}[g(r) \ln (g(r))-(g(r)-1)] r \mathrm{~d} r,
$$

where $g(r)$ is the radial distribution function. To test the $S_{2}$ freezing criterion using our data, we first computed the radial distribution functions for different area fractions,

(a)
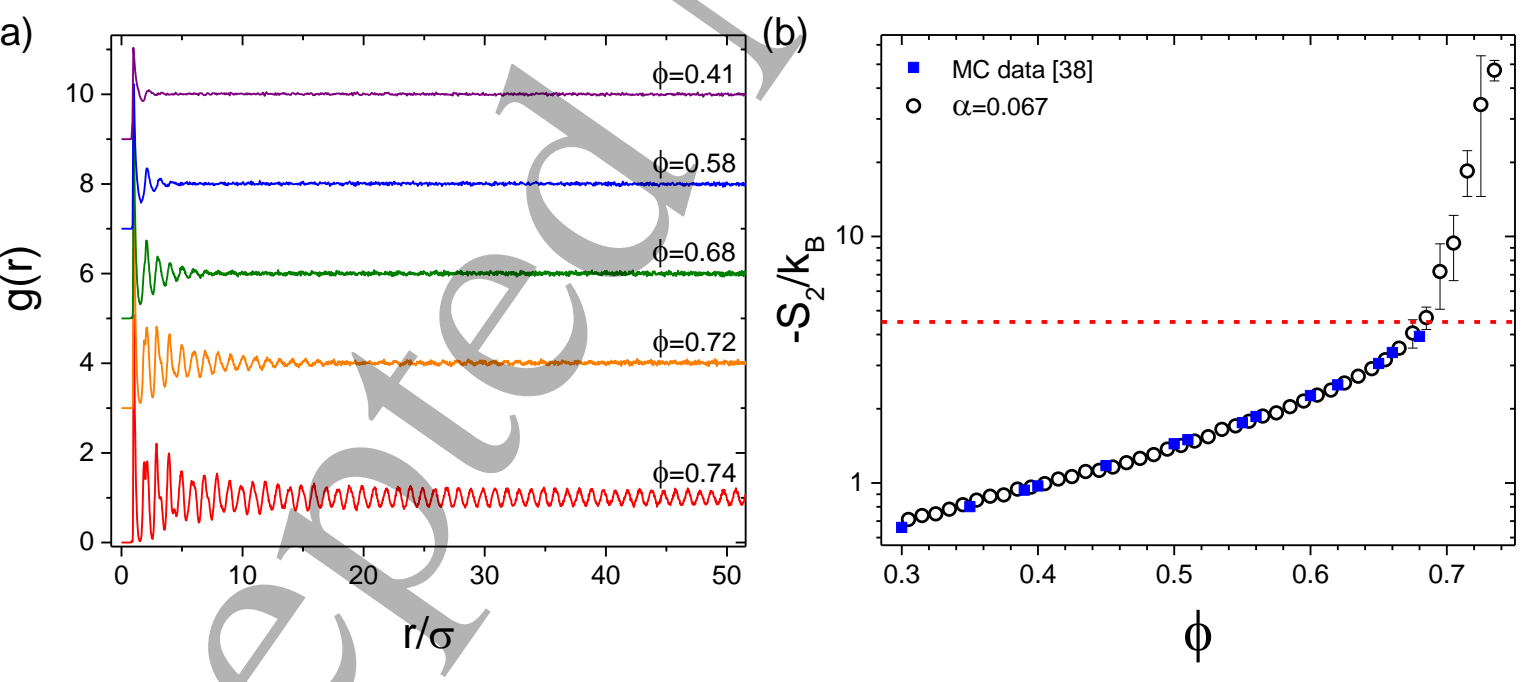

Figure 4. (a) Radial distribution functions, $g(r)$, for area fractions across the liquid, hexatic and crystal phases. Note that they are shifted vertically for clarity. (b) The excess entropy $-S_{2} / k_{B}$, calculated from the $g(r)$ via Eq. (7), as a function of the area fraction $\phi$ for $\alpha=0.067^{\circ}$. The solid line is the melting criterion of $-S_{2} / k_{B}=4.5$ [42] and blue data points show values of $-S_{2} / k_{B}$ from MC simulation from [38]. 
which are shown in Fig. 4a for area fractions spanning the liquid, hexatic and crystal phases. At area fractions of $0.41,0.58$ and 0.68 only a small number of peaks are observed, and the structure in $g(r)$ decays rapidly, characteristic of the short ranged translational order in the liquid. For the $g(r)$ in the hexatic phase $(\phi=0.72)$, the range of correlations increases to about $15 \sigma$, albeit still being short ranged, while at $\phi=0.74$ long range correlations in the $g(r)$ are observed, reflecting the quasi-long ranged translational order in the crystal.

Next, we calculated the excess entropy according to Eq. (7) for a range of area fractions as shown in Fig. 4b. At low area fractions, $-S_{2} / k_{B}$ increases roughly exponentially with $\phi$ in agreement with previous reports [38, 44] and is in good agreement with previously repported simulation results for $-S_{2} / k_{B}$ in the liquid phase [38]. Around $\phi=0.65-0.69$, however, there is a marked change in slope, which coincides with the start of the liquid-hexatic coexistence region. In [42] it was reported that at the melting transition $-S_{2} / k_{B}$ takes the value of 4.5 , which is indicated by the dashed horizontal line in Fig. 4b. Our data reach this value at an area fraction of just over $\phi=0.69$, i.e. just after the change in slope, which approximately corresponds to the start of the hexatic phase at $\phi \approx 0.70$. This behaviour is very similar to results in [42] for experiments on NIPA particles and simulations of hard disks across the freezing transition, suggesting that the value of $S_{2}$ can be used to indicate the position of the freezing transition.

\section{Conclusion}

In summary, we have further elucidated the nature of melting in two-dimensional hard spheres by studying three additional quantitiés known to show characteristic behaviour close to the liquid-hexatic and hexatic-crystal transitions. In particular, we first consider the bond-orientational correlation time, as the hexatic phase is approached from the liquid. We find it shows evidence of a divergence associated with the onset of critical fluctations, but that this is preempted by the weakly first order liquid-hexatic transition. We also compute Frank's constant, which in the hexatic phase is found to diverge upon approaching the crystal. Finally, we obtain the excess entropy from the calculation of the radial distribution functions for a wide range of area fractions across the liquid, hexatic and crystals phases and find that $-S_{2} / k_{B}$ appears to reach a characteristic value of 4.5 at the onset of the freezing transition in agreement with previous reports.

\section{Acknowledgements}

We thank Daan Frenkel, Marjolein Dijkstra, Rene van Roij, Bob Evans, Hartmut Löwen and Francois Lavergne for useful discussions. The EPSRC and the European Research Council (ERC) are acknowledged for financial support (ERC Starting Grant 279541IMCOLMAT). 
Bond-orientational order and Frank's constant in two-dimensional colloidal hard spheres9

\section{References}

[1] N. D. Mermin and H. Wagner. Absence of ferromagnetism or antiferromagnetism in one-or twodimensional isotropic Heisenberg models. Phys. Rev. Lett., 17(22):1133, 1966.

[2] Q. H. Wei, C. Bechinger, and P. Leiderer. Single-file diffusion of colloids in one-dimensional channels. Science, 287(2):625, 2000.

[3] R. Di Leonardo, S. Keen, F. Ianni, J. Leach, M. Padgett, and G. Ruocco. Hydrodynamic interactions in two dimensions. Physical Review E, 78(3):031406, sep 2008.

[4] P. N. Pusey and W. Van Megen. Phase behaviour of concentrated suspensions of nearly hard colloidal spheres. Nature, 320(27):340, 1986.

[5] J. M. Kosterlitz and D. J. Thouless. Ordering, metastability and phase transitions, in twodimensional systems. J. Phys. C: Solid State Phys., 6:1181, 1973.

[6] B. I. Halperin and D. R. Nelson. Theory of two-dimensional melting. Phys. Rev. Lett., 41:121, 1978.

[7] D. R. Nelson and B. I. Halperin. Dislocation-mediated melting in two dimensions. Phys. Rev. B, 19:2457, 1979.

[8] A. P. Young. Melting and the vector Coulomb gas in two dimensions. Phys. Rev. B, 19:1855, 1979.

[9] K. Zahn, R. Lenke, and G. Maret. Two-Stage Melting of Paramagnetic Colloidal Crystals in Two Dimensions. Phys. Rev. Lett., 82(13):2721, 1999.

[10] D. R. Nelson. Defects and geometry in condensed matter physics. Cambridge University Press, 2002.

[11] P. Keim, G. Maret, and H. H. von Grünberg. Frank's constant in the hexatic phase. Phys. Rev. E, 75(3):031402, 2007.

[12] K. Zahn and G. Maret. Dynamic criteria for melting in two dimensions. Phys. Rev. Lett., 85(17):3656, 2000.

[13] Y. Han, N. Y. Ha, A. M. Alsayed, and A. G. Yodh. Melting of two-dimensional tunable-diameter colloidal crystals. Phys. Rev. E, 77(4):041406, 2008.

[14] C. A. Murray. Experimental Studies of Melting and Hexatic Order in Two-Dimensional Colloidal Suspensions, pages 137-215. Springer New York, New York, NY, 1992.

[15] B. J. Alder and T. E. Wainwright. Phase transition in elastic disks. Phys. Rev., 127(2):359, 1962.

[16] P. Bladon and D. Frenkel. Dislocation unbinding in dense two-dimensional crystals. Phys. Rev. Lett., 74(13):2519, 1995.

[17] E. P. Bernard and W. Krauth. Two-Step Melting in Two Dimensions: First-Order Liquid-Hexatic Transition. Phys. Rev. Lett., 107(15):155704, 2011.

[18] S. C. Kapfer and W. Krauth. Two-Dimensional Melting: From Liquid-Hexatic Coexistence to Continuous Transitions. Phys. Rev. Lett., 114(3):035702, 2015.

[19] J. A. Anderson, J. Antonaglia, J. A. Millan, M. Engel, and S. C. Glotzer. Shape and symmetry determine two-dimensional melting transitions of hard regular polygons. Phys. Rev. X, 7(2):1$14,2017$.

[20] S. Deutschländer, T. Horn, H. Löwen, G. Maret, and P. Keim. Two-Dimensional Melting under Quenched Disorder. Phys. Rev. Lett., 111(9):098301, 2013.

[21] W. Qi and M. Dijkstra. Destabilisation of the hexatic phase in systems of hard disks by quenched disorder due to pinning on a lattice. Soft Matter, 11:2852, 2015.

[22] W. Qi, A. P. Gantapara, and M. Dijkstra. Two-stage melting induced by dislocations and grain boundaries in monolayers of hard spheres. Soft Matter, 10(30):5449, 2014.

[23] H. H. von Grünberg, P. Keim, and G. Maret. Phase Transitions in Two-Dimensional Colloidal Systems. In Soft Matter, volume 3, pages 40-83. Wiley, 2007.

[24] U. Gasser, C. Eisenmann, G. Maret, and P. Keim. Melting of crystals in two dimensions. ChemPhysChem, 11:963 - 970, 2010.

[25] S. Deutschländer, A. M. Puertas, G. Maret, and P. Keim. Specific heat in two-dimensional melting. 
Bond-orientational order and Frank's constant in two-dimensional colloidal hard spheres10

Phys. Rev. Lett., 113(12):1-6, 2014.

[26] C. A. Murray and D. H. Van Winkle. Experimental observation of two-stage melting in a classical two-dimensional screened Coulomb system. Phys. Rev. Lett., 58(12):1200, 1987.

[27] A. H. Marcus and S. A. Rice. Observations of First-Order Liquid-to-Hexatic and Hexatic-to-Solid Phase Transitions in a Confined Colloid Suspension. Phys. Rev. Lett., 77(12):2577, 1996.

[28] P. Karnchanaphanurach, B. Lin, and S. A. Rice. Melting transition in a quasi-two-dimensional colloid suspension: Influence of the colloid-colloid interaction. Phys. Rev. E., 61(4):4036, 2000.

[29] Y. Peng, Z. Wang, A. M. Alsayed, A. G. Yodh, and Y. Han. Melting of Colloidal Crystal Films. Phys. Rev. Lett., 104(20):205703, 2010.

[30] J. Lee and K. J. Strandburg. First-order melting transition of the hard-disk system. Phys. Rev. $B, 46(17): 11190,1992$.

[31] J. A. Zollweg and G. V. Chester. Melting in two dimensions. Phys. Rev. B, 46(17):11186, 1992.

[32] J. F. Fernández, J. J. Alonso, and J. Stankiewicz. One-stage continuous melting transition in two dimensions. Phys. Rev. Lett., 75(19):3477, 1995.

[33] A. Jaster. Computer simulations of the two-dimensional melting transition using hard disks. Phys. Rev. E., 59(3):2594, 1999.

[34] K. Binder, S. Sengupta, and P. Nielaba. The liquid-solid transition of hard discs: first-order transition or Kosterlitz-Thouless-Halperin-Nelson-Young scenario? J. Phys.: Condens. Matter, 14(14):2323, 2002.

[35] M. Engel, J. A. Anderson, S. C. Glotzer, M. Isobe, E. P. Bernard, and W. Krauth. Hard-disk equation of state: First-order liquid-hexatic transition in two dimensions with three simulation methods. Phys. Rev. E, 87(4):042134, 2013.

[36] A. L. Thorneywork, J. L. Abbott, D. G. A. L. Aarts, and R.P. A. Dullens. Two-dimensional melting of colloidal hard spheres. Phys. Rév. E, 95:012614, 2017.

[37] A. L. Thorneywork, R. Roth, D. G. A. L. Aarts, and R. P. A. Dullens. Communication: Radial distribution functions in a two-dimensional binary colloidal hard sphere system. J. Chem. Phys., 140(16):161106, 2014.

[38] A. L. Thorneywork, R. E. Rozas, R. P. A. Dullens, and J. Horbach. Effect of hydrodynamic interactions on self-diffusion of quasi-two-dimensional colloidal hard spheres. Phys. Rev. Lett., 115:268301, 2015.

[39] J. C. Crocker and D. G. Grier. Methods of digital video microscopy for colloidal studies. J. Colloid Interface Sci., 179:298, 1996.

[40] H. Watanabe, S. Yukawa, Y. Ozeki, and N. Ito. Critical exponents of isotropic-hexatic phase transition in the hard-disk system. Phys. Rev. E, 69:045103, 2004.

[41] A. del Campo. Colloidal test bed for universal dynamics of phase transitions. Proc. Natl. Acad. Sci. USA, 112:6780, 2015.

[42] Z. Wang, W. Qi, Y Peng, A. M. Alsayed, Y. Chen, and Y. Tong, P. Han. Two features at the two-dimensional freezing transitions. J. Chem. Phys., 134:034506, 2011.

[43] A. Baranyai and D. J. Evans. Direct entropy calculation from computer simulation of liquids. Physical review. A, 40(7):3817-3822, oct 1989.

[44] X. Ma, W. Chen, Z. Wang, Y. Peng, Y. Han, and P. Tong. Test of the universal scaling law of diffusion in colloidal monolayers. Phys. Rev. Lett., 110:078302, 2013. 